\title{
Some Results on how to solve uncertain transport Problems
}

\author{
Xiaoxu $\mathrm{Lu}^{1}$, Yunfei $\mathrm{Li}^{2}$ and Jintao Meng ${ }^{1, *}$ \\ ${ }^{1}$ Zhengzhou Institute of Aeronautical Industry Management, Zhengzhou, 450015, China. \\ ${ }^{2}$ Henan Procuratorial Vocational College, Zhengzhou, 450000, China. \\ *icomeon2003@126.com
}

Keywords: assignment problem; maximum element algorithm; supply and demand balance; transport problem, uncertain data.

\begin{abstract}
Assignment Problem is a key issue in optimization problem since it has large application. A lot of researchers have focused on this issue and at the same time many related problems are considered, such as traffic problems and transport problems etc. In paper, the authors considered the assignment Problem using the Maximum Element Algorithm. It has the facial application background since each manager wants to obtain the more revenue. And in the same paper they proposed that if the data is uncertain, how to solve the problem. It is fitful to the facial problems of transportation problem. We want to use the maximum element algorithm to find the scheme of the uncertain data. Since it is uncertain data we have to obtain the upper bound and lower bound. Finally we find that the Maximum Element Algorithm is a useful method to solve some optimization problems.
\end{abstract}

\section{Introduction}

Robot World Cup [1][2] is a form of football, through the competition to test new technologies in order to promote artificial intelligence, robotics and development projects of related areas; meanwhile, it also provides a broad platform to the robot hardware, software technology research. Robot soccer game is put forward in recent years of multi-agent system development platform, and it is a typical multi-agent robotic System. Soccer robot design has become the artificial intelligence and robotics research in the one of the hotspots field [3].

Transport problem is an important branch in assignment problem. There are lots of experts considered the related problems. The great part of papers are considered the minimum cost of transport problems in literatures [1,2,3,4,5]. It meets the real meaning of practical problems, where the cost of transportation is considered.

The tradition transportation problem is considered there are m suppliers A1, A2, ---,Am and the corresponding products are a1, a2, ---,am, at the same time there are $\mathrm{n}$ dealers B1, B2, ---, Bn and the corresponding demands are b1, b2, ---, bn. Let cij be the cost of transportation from Ai to $\mathrm{Bj}$ and rij be the income of the transportation from $\mathrm{Ai}$ to $\mathrm{Bj}$, where we know that different areas have different income even for the same product. We can show that relation by the following list (see the table 1).

Table 1

\begin{tabular}{|l|l|l|l|l|l|}
\hline & $\mathrm{B}_{1}$ & $\mathrm{~B}_{2}$ & $\cdots$ & $\mathrm{B}_{\mathrm{n}}$ & product \\
\hline $\mathrm{A}_{1}$ & $\mathrm{r}_{11}, \mathrm{c}_{11}$ & $\mathrm{r}_{12}, \mathrm{C}_{12}$ & $\cdots$ & $\mathrm{r}_{1 \mathrm{n}}, \mathrm{c}_{1 \mathrm{n}}$ & $\mathrm{a}_{1}$ \\
\hline $\mathrm{A}_{2}$ & $\mathrm{r}_{21}, \mathrm{c}_{21}$ & $\mathrm{r}_{22}, \mathrm{C}_{22}$ & $\cdots$ & $\mathrm{r}_{2 \mathrm{n}}, \mathrm{c}_{2 \mathrm{n}}$ & $\mathrm{a}_{2}$ \\
\hline$\cdots$ & $\cdots$ & $\cdots$ & $\cdots$ & $\cdots$ & $\cdots$ \\
\hline $\mathrm{A}_{\mathrm{m}}$ & $\mathrm{r}_{\mathrm{m} 1}, \mathrm{C}_{\mathrm{m} 1}$ & $\mathrm{r}_{\mathrm{m} 2}, \mathrm{C}_{\mathrm{m} 2}$ & $\cdots$ & $\mathrm{r}_{\mathrm{mn}}, \mathrm{C}_{\mathrm{mn}}$ & $\mathrm{a}_{\mathrm{m}}$ \\
\hline demand & $\mathrm{b}_{1}$ & $\mathrm{~b}_{2}$ & $\cdots$ & $\mathrm{b}_{\mathrm{n}}$ & \\
\hline
\end{tabular}

Normally we assume that

$$
\sum_{i=1}^{m} a_{i}=\sum_{j=1}^{n} b_{j}
$$


The problem is how to assign the transportation $\mathrm{x}_{\mathrm{ij}}$ meet the sum demand of dealers and attain the maximum benefit? We can modify the problem by the linear program:

$$
\begin{aligned}
& \max \sum_{i=1}^{m} \sum_{j=1}^{n}\left(r_{i j}-c_{i j}\right) x_{i j} \\
& \max \sum_{i=1}^{m} \sum_{j=1}^{n} d_{i j} x_{i j} \\
& \text { s.t. } \quad \sum_{j=1}^{n} x_{i j}=a_{i}, i=1, \cdots, m \\
& \text { (1) s.t. } \sum_{j=1}^{n} x_{i j}=a_{i}, i=1, \cdots, m \\
& \sum_{i=1}^{m} x_{i j}=b_{j}, j=1, \cdots, n \\
& \sum_{i=1}^{m} x_{i j}=b_{j}, j=1, \cdots, n \\
& x_{i j} \geq 0, r_{i j} \geq c_{i j} \geq 0, i=1, \cdots, m, j=1, \cdots, n \text {. } \\
& x_{i j} \geq 0, d_{i j} \geq 0, i=1, \cdots, m, j=1, \cdots, n .
\end{aligned}
$$

We can simplify the objective function by introducing a new variable function:

$$
d_{i j}=r_{i j}-c_{i j} \text {, where } d_{i j} \geq 0 \text {. }
$$

It is easy to see that $\mathrm{d}_{\mathrm{ij}}$ can be obtained by a subtraction of rij and cij. At same time we can simply the model by the following model (2).

\section{Maximum Element Algorithm}

Definition 2.1 We call transportation series such as the path $x_{i_{1} j_{1}}, x_{i_{1} j_{2}}, x_{i_{2} j_{2}}, \cdots, x_{i_{s} j_{s}}, x_{i_{s} j_{1}}$ closed path.

Definition 2.2 If a vertex is a unique vertex in the line or the column of a vertex set $x_{i_{1} j_{1}}, X_{i_{2} j_{2}}, \cdots, x_{i_{s} j_{s}}$, then we call the vertex is isolated vertex in the vertex set.

For the model (2) we want to find the maximum benefit from the transportation problem. Therefore we should assign the maximal transportation flow on the maximal $\mathrm{d}_{\mathrm{ij}}$. Hence we find $\max _{i, j} d_{i j}=d_{p q}$.

For $\mathrm{d}_{\mathrm{pq}}$ we use Maximum Element Algorithm:

Case 1 if $a_{p}<b_{q}$, then we delete the p-th line. It means that the other transportation in p-th line except $\mathrm{x}_{\mathrm{pq}}$ are zero. And let $x_{p q}:=a_{p}, b_{q}:=b_{q}-a_{p}$;

Case 2 if $b_{q}<a_{p}$, then we delete the q-th column, it means that the other transportation in q-th column except $\mathrm{x}_{\mathrm{pq}}$ are zero. And let $x_{p q}:=a_{p}, a_{p}:=a_{p}-b_{q}$;

Case 3 if $a_{p}=b_{q}$, then we delete either the q-th column or p-th line, it means that the other transportation in q-th column or in $\mathrm{p}$-th line except $\mathrm{x}_{\mathrm{pq}}$ are zero. And let $x_{p q}:=a_{p}=b_{q}, a_{p}:=0$.

For $\max _{i, j}\left\{d_{i j}-d_{p q}\right\}$ we also use the Maximum Element Method again.

Doing the Algorithm limited times we can get the following cases:

Case 1 if there is only one position retained, then we choose the fit transportation since the real products are equal to the total requirements;

Case 2 if there is one column or line retained, then we use the remaining transportation to fix the $\mathrm{x}_{\mathrm{ij}}$. We could not delete the column or the line.

Theorem [6] If $\sum_{i=1}^{m} a_{i}=\sum_{j=1}^{n} b_{j}$, then we can find the optimal solution of model (2) using the maximum element algorithm.

By the maximum element algorithm we can consider the uncertain value cases of transportation problem. Actually some suppliers can not keep the product as the same quantity. There are some factors to affect the product such as cliamte, resource constrains, work efficiency etc. The product of the i-th supplier is $\left[\mathrm{s}_{\mathrm{i}}, \mathrm{s}_{\mathrm{i}}{ }_{\mathrm{i}}\right.$. And we may assume that $\sum_{i=1}^{m} s_{i} \leq \sum_{j=1}^{n} b_{j} \leq \sum_{i=1}^{m} s_{i}^{\prime}$. Therefore we can use the method of example 2 and 3 to find the lower bound and upper bound of the optimal objective value.

Similarly the dealers' demands are also influence by some factors such as the trend, climate, and 
operating costs etc. The demand of the $\mathrm{j}$-th is $\left[\mathrm{t}_{\mathrm{j}}, \mathrm{t}_{\mathrm{j}}{ }_{\mathrm{j}}\right.$. And we may assume that $\sum_{j=1}^{n} t_{j} \leq \sum_{i=1}^{m} a_{i} \leq \sum_{j=1}^{n} t_{j}$. We also can use the former models to solve the new problems.

We can also consider that both product and demand are uncertain. It is more complex than the former models. We can use the algorithm to find some useful bound. We may consider the demand and product are random, which we know the distribution's function. We can consider mathematical expectation of the supplied products and demand. By using the algorithm, we can solve the expectation of the optimal solution and objective value' upper bound and lower bound.

\section{Some related models}

Example 1 Assume that there is a transportation problem, where $\sum_{i=1}^{m} a_{i}=\sum_{j=1}^{n} b_{j}$.

See the following table 2

Table 2

\begin{tabular}{|l|l|l|l|l|}
\hline & $\mathrm{B}_{1}$ & $\mathrm{~B}_{2}$ & $\mathrm{~B}_{3}$ & product \\
\hline $\mathrm{A}_{1}$ & 4,3 & 5,3 & 6,4 & 1 \\
\hline $\mathrm{A}_{2}$ & 5,2 & 4,3 & 4,1 & 2 \\
\hline $\mathrm{A}_{3}$ & 4,2 & 6,3 & 2,1 & 4 \\
\hline demand & 1 & 2 & 4 & \\
\hline
\end{tabular}

We first compute the $\left(\mathrm{d}_{\mathrm{ij}}\right)$, and use the maximum element algorithm to find the optimal solution.

\begin{tabular}{|l|l|l|l|l|}
\hline & $\mathrm{B}_{1}$ & $\mathrm{~B}_{2}$ & $\mathrm{~B}_{3}$ & product \\
\hline $\mathrm{A}_{1}$ & $(1), \mathrm{X}$ & $(2), \mathrm{X}$ & $(2), 1$ & 1 \\
\hline $\mathrm{A}_{2}$ & $(3), 1$ & $(1), \mathrm{X}$ & $(3), 1$ & $2,(1)$ \\
\hline $\mathrm{A}_{3}$ & $(2), \mathrm{X}$ & $(3), 2$ & $(1), 2$ & $4,(2)$ \\
\hline demand & 1 & 2 & $4,(1)$ & \\
\hline
\end{tabular}

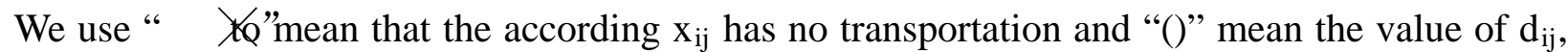
etc.

The optimal solution is $x_{2,1}=x_{2,3}=x_{1,3}=1, \quad x_{3,2}=x_{3,3}=2$.

And the optimal objective function' value is 16 .

Example 2 Under the same condition of example 1, we change the products. If the sum of product is more than the sum of demand, then we can change the model (2). Then we add an new dealer and the demand is $\sum_{i=1}^{m} a_{i}-\sum_{j=1}^{n} b_{j}$.

Let $a_{1}=2, a_{2}=3, a_{3}=5$. Then we add a demander $B_{4}$ and $d_{i, 4}=0$ for $i=1,2,3$.

\begin{tabular}{|l|l|l|l|l|l|}
\hline & $\mathrm{B}_{1}$ & $\mathrm{~B}_{2}$ & $\mathrm{~B}_{3}$ & $\mathrm{~B}_{4}$ & product \\
\hline $\mathrm{A}_{1}$ & $(1), \mathrm{X}$ & $(2), \mathrm{X}$ & $(2), 2$ & $(0), 0$ & 2 \\
\hline $\mathrm{A}_{2}$ & $(3), 1$ & $(1), \mathrm{X}$ & $(3), 2$ & $(0), 0$ & 3 \\
\hline $\mathrm{A}_{3}$ & $(2), \mathrm{X}$ & $(3), 2$ & $(1), 0$ & $(0), 3$ & 5 \\
\hline demand & 1 & 2 & 4 & 3 & \\
\hline
\end{tabular}

The optimal solution is $x_{2,1}=1, x_{3,4}=3, x_{3,2}=x_{1,3}=x_{2,3}=2$.

And the optimal objective function' value is 19 .

Example 3 Under the same condition of example 1, we change the demands. If the sum of product is less than the sum of demand, then we add an new supplier and the product is $\sum_{j=1}^{n} b_{j}-\sum_{i=1}^{m} a_{i}$.

Let $b_{1}=2, b_{2}=2, b_{3}=4$. Then we add a producer $A_{4}$ and $d_{4, j}=0$ for $j=1,2,3$. 


\begin{tabular}{|l|l|l|l|l|}
\hline & $\mathrm{B}_{1}$ & $\mathrm{~B}_{2}$ & $\mathrm{~B}_{3}$ & product \\
\hline $\mathrm{A}_{1}$ & $(1), \mathrm{X}$ & $(2), \mathrm{X}$ & $(2), 1$ & 1 \\
\hline $\mathrm{A}_{2}$ & $(3), 1$ & $(1), \mathrm{X}$ & $(3), 1$ & 2 \\
\hline $\mathrm{A}_{3}$ & $(2), \mathrm{X}$ & $(3), 2$ & $(1), 2$ & 4 \\
\hline $\mathrm{A}_{4}$ & $(0), 1$ & $(0), 0$ & $(0), 0$ & 1 \\
\hline demand & 2 & 2 & 4 & \\
\hline
\end{tabular}

And the optimal objective function' value is 16 .

Example 3 Under the same condition of example 1, we have the products is uncertain. Let $a_{i} \in\left[\mathrm{s}_{i}, \mathrm{~s}_{i}^{\prime}\right]$. We combine the example 1 and example 2. Let $a_{1} \in[1,2], a_{2} \in[2,3], a_{3} \in[4,5]$ and $b_{1}=1, b_{2}=2, b_{3}=4$. By the above method, we have the optimal value is between 16 and 19 .

\section{Summary}

We consider the uncertain data of the transportation problem. Using the Maximum Element Algorithm, we the optimal value' upper bound and lower bound. By similar way, we can consider the uncertain demand data. Even we can consider the uncertain demand and product data by the similar way. Furthermore, we can use random method to consider the uncertain data.

\section{Acknowledgement}

This research was financially supported by the Chinese National Natural Science Youth Foundation (Project No. 11401605) and the Key Scientific Research Project of Higher School Education Department of Henan Province (Project No. 14A110027).

\section{References}

[1] Qian Songdi, Operational Research (3-th edition). Tsinghua University Press, 2005.

[2] Xie Youcai, "A Network Algorithm of the Transportation Problem of the Shortest Time Limit and Its Additional Information”, Operations Research and Management Science 2003, 12(6):62-66.

[3] Li Zhenping, "Transportation problem to the shortest time limit and its algorithm on graph”, Operations Reserch and Management Science1999, 8(4):6631-6636.

[4] Bai Guozhong, "B-assignment Problem”, Journal of systems science and systems engineering 1997, 6 (1) :1-4,.

[5] Hu Xiaodong and Zhang Haisheng, “Applying hierarchy model to multi-objective decision making on postal transportation operations”, Systems engineering and Electronics, 2001

[6] Shangwu Yang and Jing Li, “Some results on how to solve transport problems” , submitted. 